\title{
DETECÇÃO DE FALTAS EVOLUTIVAS E MÚLTIPLOS DISTÚRBIOS EM REGISTROS OSCILOGRÁFICOS BASEADA NA TRANSFORMADA WAVELET DISCRETA
}

\author{
Flávio Bezerra Costa* \\ flabclee.ufcg.edu.br
}

\author{
Benemar Alencar de Souza* \\ benemardee.ufcg.edu.br
}

\author{
Núbia Silva Dantas Brito* \\ nubia@dee.ufcg.edu.br \\ * Universidade Federal de Campina Grande - CEEI/UAEE/GSE \\ Av. Aprígio Veloso 882, Bodocongó \\ 58109-970 Campina Grande - Brasil
}

\section{RESUMO}

Apresenta-se neste artigo um método de detecção automática de faltas baseado na análise da energia janelada dos coeficientes wavelet das tensões e correntes de fase. O método é capaz de detectar faltas evolutivas e de múltiplos distúrbios transitórios, a exemplo de uma falta seguida por um religamento automático, capturados no mesmo registro oscilográfico. O método também consegue distinguir faltas de algumas ocorrências relacionadas à qualidade da energia elétrica, tais como afundamento de tensão e transitórios de chaveamento. O método é eficaz na identificação dos instantes inicial e final da falta, além de conseguir analisar sinais referentes a linhas de transmissão de diferentes classes de tensão e que tenham sido registrados com frequências de amostragem diversas. Em avaliação de desempenho com registros oscilográficos simulados e reais, o método apresentou bons resultados.

PALAVRAS CHAVES: Falta evolutiva, registros com múltiplos distúrbios, transformada wavelet.

\section{ABSTRACT}

Detection of Crosscountry Fault and Multiple Distur-

Artigo submetido em 11/05/2009 (Id.: 01003)

Revisado em 01/08/2009, 05/10/2009

Aceito sob recomendação do Editor Associado Prof. Ivan Nunes Da Silva bances In Oscillographic Data Based On Wavelet Transform

This paper presents an automatic fault detection method based on the analysis of the wavelet coefficient energies of the phase voltages and currents. The method detects developing faults and multiple transient disturbances such as a fault followed by an automatic line reclosing in a single oscillographic record. The method also sort fault from some power quality disturbances such as voltage sags and switching transients. The times regarding the fault inception and clearance are also identified. Records from a nonspecific line in a generic network with different sampling rates and rated voltages can be evaluated without distinction. The performance of the method was evaluated for both actual and simulated data and good results were obtained.

KEYWORDS: Developing fault, records with multiple disturbances, wavelet transform.

\section{INTRODUÇÃO}

A Agência Nacional de Energia Elétrica (ANEEL) utiliza alguns indicadores para verificar a qualidade dos serviços públicos de energia elétrica no Brasil. No caso particular dos sistemas de transmissão de energia elétrica, a qualidade do serviço é medida com base na disponibilidade e na capacidade plena de seu sistema (linhas de transmissão (LTs) e demais equipamentos), sendo este considerado in- 
disponível quando ocorre desligamento programado ou nãoprogramado (ANEEL, 2007).

As faltas em sistemas elétricos de potência são desligamentos não programados de seus componentes, originadas das condições adversas as quais o sistema está sempre submetido. Na prática, as faltas são ocasionadas por rompimentos dos cabos em LTs, falhas em equipamentos e fenômenos naturais, tais como: descargas atmosféricas, acumulação de poeira em isoladores, ventos fortes e queimadas. Após a ocorrência e eliminação de uma falta, uma análise mais detalhada do evento é imprescindível para avaliação e correção dos sistemas de proteção, avaliação do desempenho dos disjuntores, confirmação das ocorrências e diagnóstico das faltas. Desta forma, medidas mitigadoras podem ser tomadas, resultando em melhorias na qualidade dos serviços públicos de energia elétrica.

Os registradores digitais de perturbação (RDPs) coletam dados do sistema, armazenando-os em registros oscilográficos, os quais são posteriormente transferidos ao servidor central da oscilografia para serem analisados por técnicos especializados. Os registros podem conter informações sobre faltas e distúrbios de qualidade da energia elétrica (QEE). Entretanto, muitas vezes não contêm nenhuma informação de destaque. O grande volume de dados coletados causa problemas de armazenamento e de tráfego na rede de oscilografia (Silva et al, 2007). Para reduzir tais problemas, empresas como a Chesf (Companhia Hidro-Elétrica do São Francisco) vêm investindo na automação de diagnóstico de faltas em que sejam evitados a transferência de registros sem relevância para o servidor central da oscilografia.

Dentre as diversas técnicas utilizadas no diagnóstico de distúrbios transitórios em sistemas elétricos de potência, destaca-se a transformada wavelet discreta (TWD). Robertson et al. (1996) e Oleskovicz et al. (2006) apresentaram estudos comparativos entre a TWD e outras técnicas, mostrando exemplos de aplicação e as vantagens da utilização da TWD na extração de características e análise de distúrbios transitórios. Bollen and $\mathrm{Gu}$ (2006) apresentaram um exemplo prático para detecção e localização dos instantes inicial e final de um afundamento de tensão. Costa et al. (2006a) propôs um método baseado na TWD para localização no tempo dos instantes inicial e final de faltas. Santoso et al. (1996) e Costa et al. (2008) reportaram diferentes metodologias baseadas na TWD para detecção e classificação de distúrbios de QEE. Recentemente, muitos métodos de classificação de faltas baseados na TWD também vêm sendo propostos na literatura (Silveira et al., 2002; Costa et al., 2006b; Costa et al., 2006c).

Silva et al. (2007) propuseram um método de detecção e classificação de faltas em LTs baseado nas redes neurais artifici- ais (RNAs) e na TWD. O método foi utilizado, em escala piloto, em uma LT de uma das subestações da Chesf e bons resultados foram reportados. Após a sua utilização prática, algumas limitações foram identificadas, na qual destaca-se a incapacidade de análise de registros oscilográficos com faltas evolutivas e com múltiplos distúrbios, a exemplo de uma falta seguida por um religamento automático. Com relação às RNAs, a principal dificuldade prática consiste na utilização do método para analisar dados oscilográficos provenientes de outras LTs, caso seja necessária a formação de uma nova base de dados e a realização de um novo treinamento para reajuste dos parâmetros da RNA.

Visando superar algumas deficiências do método proposto por Silva et al. (2007) e atender às necessidades da identificação e transferência automática de registros oscilográficos com faltas ao servidor central da oscilografia, um novo método de diagnóstico de faltas é proposto neste artigo (Fig. 1). O método é baseado unicamente na utilização da TWD e utiliza as energias janeladas dos coeficientes wavelet da primeira escala (primeiro detalhe) das tensões e correntes. Além das faltas, o método proposto permite a detecção de algumas ocorrências relacionadas à QEE, tais como afundamentos de tensão e transitórios de chaveamento. Conforme a Fig. 1, após a detecção dos distúrbios o método localiza os respectivos instantes inicial e final das ocorrências. No caso de faltas, tem-se a classificação do curto-circuito. A principal contribuição do método proposto é a análise das faltas evolutivas e múltiplos distúrbios. As etapas de detecção e localização dos instantes de falta serão detalhadas neste artigo.

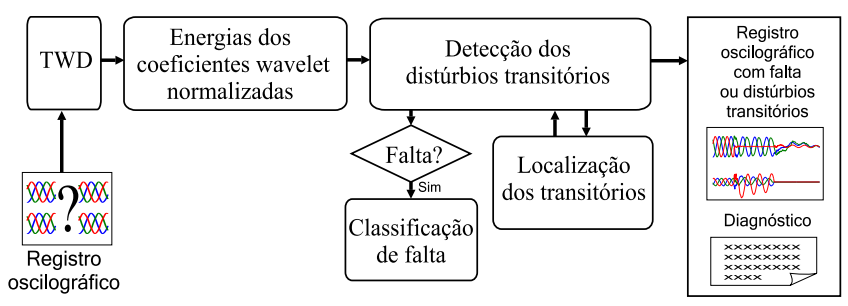

Figura 1: Método de diagnóstico de distúrbios transitórios.

O desempenho do método proposto foi avaliado com registros reais e simulados. Os registros reais foram obtidos em várias LTs do sistema Chesf, com níveis de tensão: 138, 230 e $500 \mathrm{kV}$ e taxa de amostragem de 1,2 ou 15,36 kHz. Os resultados obtidos atestaram uma boa generalização do método na análise de distúrbios transitórios do sistema Chesf, visto que bons resultados foram obtidos independente do nível de tensão e taxa de amostragem dos registros oscilográficos.

Apresenta-se também neste artigo uma análise de registros oscilográficos reais com faltas, revelando alguns aspectos dos fenômenos transitórios e os respectivos efeitos nos coeficientes wavelet que, muitas vezes, não são encontrados em registros simulados. 

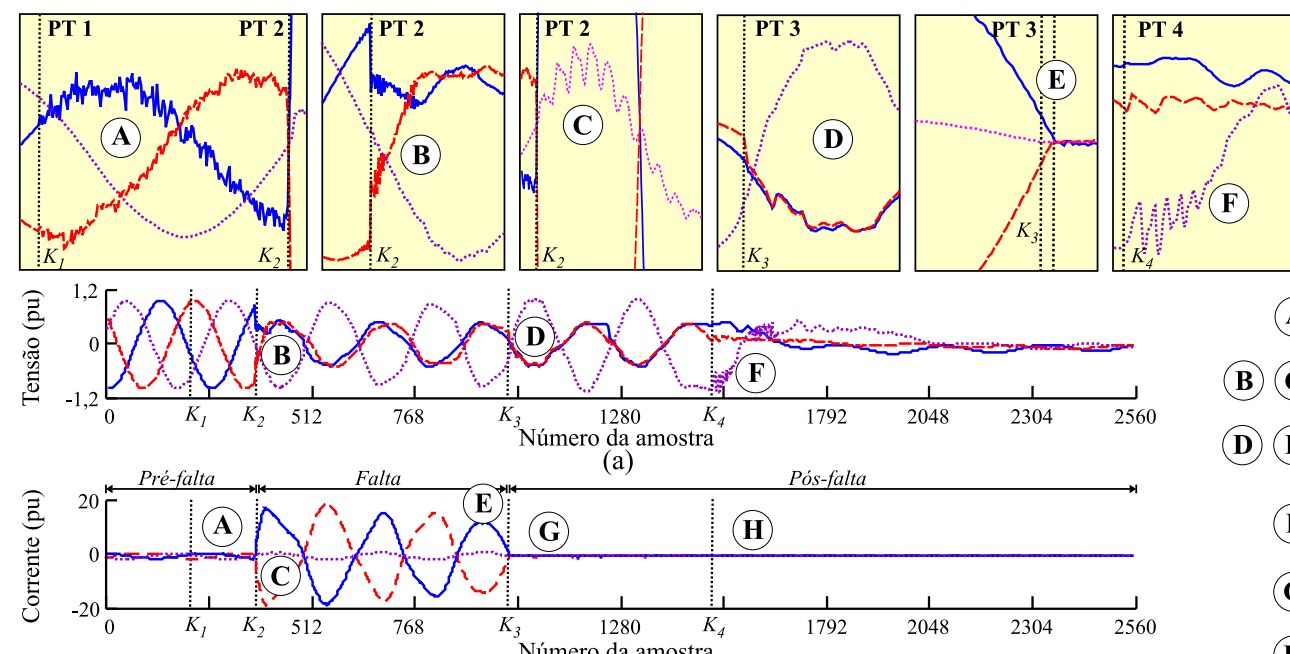

(b)
- fase A --- fase B ........ fase C

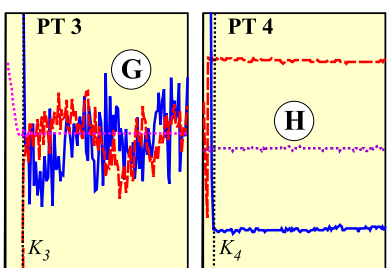

Transitórios pré-falta nas correntes de falta

B C) Transitórios de falta

D E Transitórios na eliminação da falta (terminal local)

F Transitórios na eliminação da falta (terminal remoto)

G Transitórios pós-falta nas correntes de falta

H Ruídos pós-falta nas correntes

Figura 2: Registro oscilográfico real de uma falta $A B$ : (a) tensões; (b) correntes.

\section{DISTÚRBIOS TRANSITÓRIOS}

Distúrbios em registros que apresentam fenômenos transitórios, tais como as faltas e transitórios de chaveamento são denominados neste artigo de distúrbios transitórios.

\subsection{Faltas}

Na Fig. 2 apresentam-se as tensões e correntes de fase, em p.u., de um registro oscilográfico real com uma falta bifásica do tipo AB. De um modo geral, em uma situação de falta identificam-se três períodos distintos:

1. Período de pré-falta: compreende as amostras dos sinais referentes à situação de regime permanente do sistema elétrico, antes da ocorrência da falta;

2. Período de falta: corresponde ao período no qual o sistema encontra-se em uma situação de falta. Dependendo da natureza da falta e das condições elétricas do sistema, é comum o surgimento de um aumento nas correntes e um afundamento nas tensões das fases envolvidas no curto-circuito (Bollen, 2000);

3. Período de pós-falta: contém as amostras dos sinais após a atuação da proteção.

A transição entre os diversos períodos se caracteriza pela presença de transitórios decorrentes do surgimento e eliminação da falta, os quais neste artigo são denominados de períodos transitórios (PTs). O $i$-ésimo PT inicia-se na amostra $k_{i}$.

Estudo minucioso de registros oscilográficos reais mostra que métodos de diagnóstico de faltas avaliados apenas com dados simulados podem apresentar limitações práticas, visto que os registros reais normalmente revelam alguns aspectos dos fenômenos transitórios dos distúrbios, dificilmente encontrados em simulações digitais. As principais características dos fenômenos transitórios observados na falta ilustrada na Fig. 2 são apresentados a seguir.

\subsubsection{Transitórios Pré-falta nas Correntes de Falta}

Análise de registros oscilográficos reais com falta mostrou, algumas vezes, a existência de oscilações de alta frequência (transitórios pré-falta) nas correntes de falta antes da ruptura dielétrica do meio isolante (Fig. 2). Não se encontrou na literatura relato de nenhuma simulação que tenha levado em conta esses transitórios pré-falta. Dependendo da taxa de amostragem dos sinais, os transitórios pré-falta podem influenciar consideravelmente os coeficientes wavelet da primeira escala, dificultando a localização precisa do instante inicial das faltas.

\subsubsection{Transitórios de Falta}

Após a ocorrência de uma falta, sucessivas reflexões de ondas viajantes na LT originam transitórios nas tensões e correntes (Swift, 1979; Girsis and Brown, 1983). Conforme ilustrado na Fig. 2, após a amostra $k_{2}$ (PT 2), o instante inicial da falta é bem caracterizado pela incidência de transitórios nas tensões e correntes.

A natureza dos transitórios de falta depende de diversos fatores, tais como o ângulo de incidência, resistência e localização da falta. $\mathrm{O}$ espectro de frequência desses transitórios foi cuidadosamente investigado por Swift (1979) e deve ser levado em consideração para identificar a escala wavelet mais apropriada à detecção desses fenômenos, conforme a taxa de amostragem dos sinais.

Além dos transitórios devido às ondas viajantes, as correntes de falta durante o curto-circuito (entre $k_{2}$ e $k_{3}$ na Fig. 2) são normalmente compostas por uma componente senoidal à frequência fundamental, alguns harmônicos e uma componente com decaimento exponencial, denominada de componente $d c$, cuja amplitude e decaimento depende de alguns fatores, tais como o ângulo de incidência e a resistência de falta (Bradley et al., 1978). 
Os transformadores de corrente (TCs) são dimensionados para suportarem as sobrecorrentes de falta, sendo atribuída à componente dc a principal causa da saturação dos TCs (Bradley et al., 1978; Kang et al., 2004), o que resulta em distorções nas correntes de falta do secundário dos TCs. Este fato pode influenciar de forma decisiva no cálculo dos coeficientes wavelet (Li et al., 2002; Hong and ChangChian, 2008).

\subsubsection{Transitórios na Eliminação da Falta (Termi- nal Local)}

Em um sistema trifásico, as correntes nas fases A, B e C não são interrompidas simultaneamente. O acoplamento eletromagnético existente entre as fases da LT induz transitórios nas tensões e correntes de fase após a abertura do primeiro pólo (Zaneta Jr., 2003). Como consequência, a localização exata dos instantes de abertura do disjuntor para conter a falta não é uma tarefa fácil.

\subsubsection{Transitórios na Eliminação da Falta (Termi- nal Remoto)}

Além da dispersão mecânica entre os contatos do disjuntor, outros fatores dificultam a identificação do instante final da falta. Os disjuntores em cada extremidade da LT podem abrir em instantes consideravelmente distintos, dependendo do esquema de proteção utilizado e do local de ocorrência da falta. A operação de um relé de distância, por exemplo, baseia-se em várias zonas de proteção. A primeira zona deve cobrir cerca de $80 \%$ da LT, sendo os $20 \%$ restantes protegidos pela zona secundária que cobre os $100 \%$ da LT e possui um atraso na atuação da ordem de 200 à 500 ms (Anderson, 1999). Considerando-se o caso prático de um relé em cada terminal da LT, observa-se que em cerca de $40 \%$ da LT, a extinção completa da falta é retardada pelo atraso de segunda zona.

A falta ilustrada na Fig. 2 ocorreu na primeira zona de um relé, localizado no terminal de monitoramento (terminal local), e na segunda zona de um outro relé, localizado no terminal remoto. $\mathrm{O}$ sistema de proteção atuou inicialmente no terminal local, abrindo o disjuntor no instante $k_{3}$. Aproximadamente 2 ciclos após $k_{3}$, o disjuntor no terminal remoto realizou uma operação de abertura em $k_{4}$.

Destacam-se na Fig. 2 os transitórios nas tensões na eliminação da fata, no instante $k_{4}$. Neste caso, o método proposto identifica os instantes referentes às duas manobras de chaveamento solicitadas pela proteção.

\subsubsection{Transitórios Pós-falta nas Correntes de Falta}

Quando o sistema de proteção atua inicialmente no terminal local e posteriormente, no terminal remoto, as correntes de falta no secundário do TC do terminal local podem apresentar transitórios até a extinção do arco elétrico na LT (Summary Update of Practices on Breaker Failure Protec- tion, 1982), como destacados na Fig. 2, entre as amostras $k_{3}$ e $k_{4}$. Esses transitórios influenciam os coeficientes wavelet da primeira escala e podem dificultar à detecção e localização dos instantes em que a falta é eliminada.

\subsubsection{Ruídos Pós-falta nas Correntes}

Além das distorções, a representação da corrente de falta no secundário do TC não possui valor nulo no instante em que a corrente do primário do TC é interrompida. Quando a corrente de falta é interrompida por um disjuntor na sua passagem por zero, a corrente de falta do secundário do TC tem um valor positivo ou negativo. Como consequência, as correntes pós-falta apresentam um decaimento exponencial em função de uma constante de tempo conforme o circuito do secundário do TC e com pequenas oscilações (Summary Update of Practices on Breaker Failure Protection, 1982). Essas características também podem influenciar os coeficientes wavelet da primeira escala.

\subsubsection{Transitórios Pós-falta nas Tensões}

Transitórios também podem ocorrer nas tensões do secundário dos transformadores de potencial capacitivos (TPCs) após a eliminação da falta (Bollen, 2000).

\subsection{Faltas Evolutivas}

Falta evolutiva é um curto-circuito que, com o passar do tempo, envolve outras fases, anteriormente sãs. Por exemplo, uma falta monofásica pode evoluir para uma falta bifásica para terra (Bollen and Gu, 2006). Apesar de ser um evento não muito comum em sistemas de transmissão, este tipo de distúrbio deve ser considerado em métodos de diagnóstico com aplicações práticas.

Em uma falta evolutiva, o instante em que a falta muda de tipo é caracterizado pela incidência de transitórios (Bollen and $\mathrm{Gu}, 2006$ ). Do ponto de vista dos transitórios, a única diferença em relação a uma falta simples é a ocorrência de um novo PT no instante em que a falta muda de tipo.

\subsection{Distúrbios e Múltiplos Distúrbios em Registros Oscilográficos}

Afundamento de tensão é um distúrbio de QEE caracterizado por uma redução do valor eficaz das tensões por período de curta duração. Em sistemas de transmissão de energia elétrica, de uma maneira geral, eles são decorrentes de faltas adjacentes. Parâmetros como amplitude e duração não caracterizam completamente os afundamentos de tensão, haja vista a presença de fenômenos transitórios em seus instantes inicial e final devida à ocorrência e eliminação das faltas (Bollen, 2000; Bollen and Gu, 2006).

Faltas, afundamentos de tensão e distúrbios transitórios de- 
vido às manobras de chaveamento, como energizações e desligamentos de LTs e de banco de capacitores (BCs) apresentam um ou mais PTs e são detectados pelo método.

Muitas faltas em LTs são temporárias. Nestes casos, o autoreligamento da LT implica em uma rápida restauração do sistema elétrico de potência. Tipicamente, o tempo morto referente ao primeiro religamento é da ordem de 0,5 a $1,5 \mathrm{~s} \mathrm{(30} \mathrm{a}$ 90 ciclos em $60 \mathrm{~Hz}$ ), mas pode ser frequentemente ajustado pelos engenheiros de planejamento (IEEE Power Engineering Society, 2003). Dados relacionados às faltas e aos religamentos em uma LT podem ser armazenados em um único registro oscilográfico (múltiplos distúrbios).

\section{ENERGIA JANELADA DOS COEFICIEN- TES WAVELET}

A transformada wavelet é uma ferramenta matemática de análise de sinais, que por meio de operações de escalonamentos e translações em uma função base (wavelet mãe), decompõe um sinal em diferentes faixas de frequências. $\mathrm{Na}$ maioria das aplicações práticas, predomina a utilização da TWD, comumente implementada na forma de um banco de filtros escalonados em oitavas (Mallat, 1989).

Aplicando-se a TWD a um sinal discreto $c_{o}$, com $k_{f}$ amostras e frequência de amostragem $f_{s}$, tem-se:

$$
\begin{aligned}
& c_{j}(k)=\sum_{n} h(n-2 k) c_{j-1}(n), \\
& d_{j}(k)=\sum_{n} g(n-2 k) c_{j-1}(n),
\end{aligned}
$$

sendo $c_{j}$ e $d_{j}$ os coeficientes de aproximação e wavelet da escala $j$, respectivamente; $h(k)$ e $g(k)$ os filtros passa-baixa e passa-alta, respectivamente.

O método proposto baseia-se no uso da energia janelada dos coeficientes wavelet da primeira escala. Em uma escala $j$, a energia janelada é calculada através de uma janela deslizante nos coeficientes wavelet, deslocando-se um coeficiente a cada passo de tempo (Costa et al., 2007; Costa et al., 2008), como segue:

$$
e_{j}(k)=\left\{\begin{array}{l}
\sum_{n=1}^{W / 2^{j}} d_{j}^{2}(n), \quad \text { se } 1 \leqslant k \leqslant \frac{W}{2^{j}} \\
\sum_{n=k-W / 2^{j}+1}^{k} d_{j}^{2}(n), \text { se } \frac{W}{2^{j}}<k \leqslant \frac{k_{f}}{2^{j}}
\end{array}\right.
$$

sendo $W$ o tamanho da janela, $k=\left\{1,2, \ldots, k_{f} / 2^{j}\right\}$ e $k_{f}$ o número de amostras do sinal original. Neste artigo, $W$ é a quantidade de amostras contidas em um ciclo à frequência fundamental $\left(W=f_{s} / f\right)$.

Um sinal discreto com taxa de amostragem $f_{s}$ possui um espectro de frequência limitado, variando de 0 a $f_{s} / 2 \mathrm{~Hz}$ (Oppenheim and Schafer, 1989). O uso de pares de filtros wavelet escalonados em oitavas divide o espectro de frequência do sinal de entrada pela metade. Como consequência, os coeficientes wavelet da primeira escala apresentam banda de passagem entre $f_{s} / 4$ e $f_{s} / 2 \mathrm{~Hz}$, ou seja, os coeficientes wavelet da primeira escala contêm as componentes de mais alta frequência do sinal original (Daubechies, 1992). Portanto, os coeficientes wavelet e suas respectivas energias janeladas, da primeira escala, são apropriados para detecção e localização das componentes de alta frequência oriundas dos transitórios em registros oscilográficos com distúrbios.

O método utiliza a wavelet Daubechies 4 (Daubechies, 1992), visto que esta wavelet mãe tem se mostrado eficiente na detecção de transitórios em sistemas de potência (Santoso et al., 1996; Bollen and Gu, 2006; Silva et al., 2007).

\subsection{Normalização das Curvas de Energia}

O método proposto utiliza um esquema de normalização aplicado às curvas de energias dos coeficientes wavelet da primeira escala, das tensões e correntes de fase $\left(e_{v_{A}}, e_{v_{B}}, e_{v_{C}}\right.$, $e_{i_{A}}, e_{i_{B}}$ e $\left.e_{i_{C}}\right)$. Com isso, registros oscilográficos provenientes de diversas LTs, com várias taxas de amostragem e diversos níveis de tensão podem ser avaliados indistintamente.

O esquema de normalização adotado baseou-se na análise das curvas de energias $e_{v_{A}}, e_{v_{B}}, e_{v_{C}}, e_{i_{A}}, e_{i_{B}}$ e $e_{i_{C}}$ obtidas de uma base de dados composta de 30 registros oscilográficos reais sem distúrbios. Nesta base de dados, entretanto, o cálculo das energias é feito com registros amostrados a 15360 $\mathrm{Hz}$, com as tensões e correntes em p.u..

As tensões e correntes desses registros, em condições de operação normal, apresentam ruídos elétricos de alta frequência com distribuição normal, os quais influenciam no cálculo das energias dos coeficientes wavelet da primeira escala. Após uma análise estatística, constatou-se que os valores de energia nos registros sem distúrbios estão localizados em uma região demarcada pelos limiares $E_{1}=1.10^{-5}$ e $E_{2}=8.10^{-5}$.

As curvas de energias dos coeficientes wavelet das tensões são normalizadas, de modo que as energias referentes ao regime permanente se localizem entre os limiares $E_{1}$ e $E_{2}$. Ao final, as energias normalizadas são definidas como segue:

$$
\begin{aligned}
& \left\{\mathcal{E}_{v_{A}}(k), \mathcal{E}_{v_{B}}(k), \mathcal{E}_{v_{C}}(k)\right\}=\frac{E_{2}-E_{1}}{2 E_{\text {med }}}\left\{e_{v_{A}}(k), e_{v_{B}}(k), e_{v_{C}}(k)\right\}, \\
& \left\{\mathcal{E}_{i_{A}}(k), \mathcal{E}_{i_{B}}(k), \mathcal{E}_{i_{C}}(k)\right\}=\frac{E_{2}-E_{1}}{2 E_{\text {medI }}}\left\{e_{i_{A}}(k), e_{i_{B}}(k), e_{i_{C}}(k)\right\},
\end{aligned}
$$

sendo $\mathcal{E}_{v_{A}}, \mathcal{E}_{v_{B}}, \mathcal{E}_{v_{C}}, \mathcal{E}_{i_{A}}, \mathcal{E}_{i_{B}}$ e $\mathcal{E}_{i_{C}}$ as energias normalizadas dos coeficientes wavelet, da primeira escala, das tensões e correntes de fase $v_{A}, v_{B}, v_{C}, i_{A}, i_{B}$ e $i_{C}$, respectivamente; $E_{\text {medV }}$ e $E_{\text {medI }}$ os valores médios de energia das tensões e correntes de fase, respectivamente, calculados em um ciclo.

Os valores $E_{m e d V}$ e $E_{m e d I}$ são calculados em um ciclo rela- 


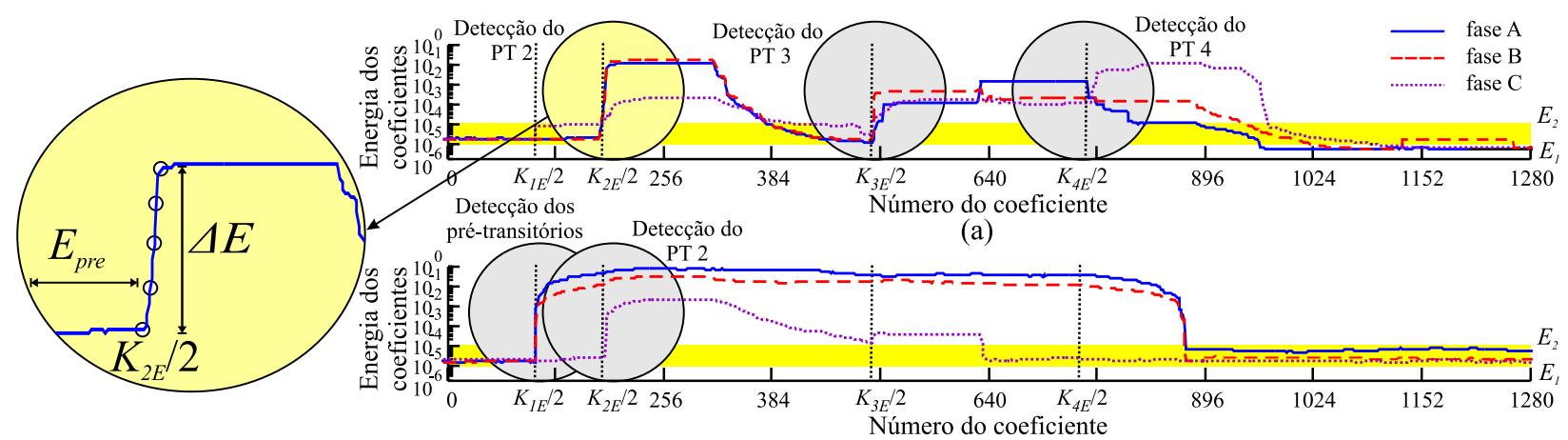

(b)

Figura 3: Energia janelada dos coeficientes wavelet da primeira escala de um registro oscilográfico real com uma falta $A B$ : (a) tensões; (b) correntes.

cionado ao regime permanente, normalmente o primeiro ciclo. Em registros oscilográficos com energização de LTs ou BCs, por exemplo, o último ciclo é utilizado para cálculo de $E_{\text {medV }}$ e $E_{\text {medI }}$.

\subsection{Comportamento das Energias}

Para ilustrar o comportamento das curvas de energias, selecionou-se o registro oscilográfico da Fig. 2, cujas energias são apresentadas na Fig. 3.

De modo geral, as curvas de energias normalizadas de registros com distúrbios transitórios apresentam magnitudes entre os limiares $E_{1}$ e $E_{2}$, nas regiões correspondentes ao regime permanente, e aumentos bruscos de energia nos instantes de ocorrência dos diversos PTs.

Os aumentos bruscos de energia não acontecem instantaneamente, principalmente em registros com transitórios amortecidos. A análise das curvas de energias de registros com distúrbios transitórios indicou que cinco amostras de energia são suficientes para detectar um PT. Considerando-se uma curva de energia genérica $(\mathcal{E})$, o método avalia progressivamente, a partir da primeira amostra $(\mathcal{E}(1))$, o aumento do valor de energia em cinco amostras consecutivas, definido a seguir:

$$
\Delta E(k)=\frac{\max \{\mathcal{E}(k), \mathcal{E}(k+1), \ldots, \mathcal{E}(k+4)\}-\mathcal{E}(k)}{\mathcal{E}(k)},
$$

sendo: $\max \{$.$\} um operador que retorna o valor máximo$ de energia nas amostras avaliadas. Considera-se $\mathcal{E}(k)=E_{1}$, quando $\mathcal{E}(k)<E_{1}$.

Neste artigo, um PT $i$, na amostra $k$ da curva de energia $\mathcal{E}$ é detectado se algum $\Delta E(k) \geqslant 3$, o que indica que as próximas amostras de energias sofrerão um aumento de no mínimo 3 vezes o valor de $\mathcal{E}(k)$ (aumento brusco de energia). O fator 3 foi determinado de forma empírica.

Além das faltas, os demais distúrbios transitórios (afundamentos de tensão, energizações e desligamentos de LTs, etc.) também apresentam variações bruscas de energia nos PTs relacionados à incidência dos transitórios. Este comportamento torna as curvas de energias uma poderosa ferramenta para detecção de distúrbios transitórios.

A detecção do $i$-ésimo PT, em uma curva de energia genérica $(\mathcal{E})$ é feita baseando-se no uso dos seguintes parâmetros:

1. $E_{1}$ e $E_{2}$ : limiares que delimitam a região referente ao regime permanente.

2. $\Delta E(k)$ : aumento de energia a partir da amostra $k$.

3. $k_{i E}$ : amostra relacionada ao instante inicial do $i$-ésimo $\mathrm{PT}\left(\Delta E(k) \geqslant 3 \Rightarrow k_{i E}=2 k\right)$.

4. $E_{\text {pre }}$ : valor médio de energia calculado em um ciclo anterior ao $i$-ésimo PT.

Além da detecção dos diversos PTs nos distúrbios, a análise das curvas de energias torna possível a localização dos instantes de falta e chaveamento, nas amostras $k_{i E}$ (Fig. 3).

Registros oscilográficos com falta seguido por religamento automático de uma LT (múltiplo distúrbios), por exemplo, apresentam PTs relacionados à falta (distúrbio 1) e PTs relacionados ao religamento automático (distúrbio 2). Portanto, é necessário identificar os PTs relacionados a cada distúrbio.

A combinação do valor médio de energia $E_{\text {pre }}$, referente a cada uma das curvas de energias $\left(\mathcal{E}_{v_{A}}, \mathcal{E}_{v_{B}}, \mathcal{E}_{v_{C}}, \mathcal{E}_{i_{A}}, \mathcal{E}_{i_{B}}\right.$ e $\mathcal{E}_{i_{C}}$ ) é utilizada para verificar se o $i$-ésimo PT pertence ou não a um determinado distúrbio. Por exemplo, no primeiro PT, $E_{p r e}<E_{2}$ em todas as curvas de energias (Fig. 3). Neste caso, o primeiro PT faz referência ao início da falta. Por outro lado, os PTs 2, 3 e 4 apresentam pelo menos um $E_{\text {pre }} \geqslant E_{2}$, indicando que estes PTs são devidos a um único distúrbio. Com relação a uma falta seguida por um religamento automático, o PT relacionado ao religamento apresentaria $E_{\text {pre }}<E_{2}$ em todas as curvas de energias, caracterizando a detecção do segundo distúrbio.

Uma vez detectados e identificados os PTs de um distúrbio, é possível através da análise das curvas de energias distinguir o evento em: falta ou demais distúrbios transitórios. Para isso, em cada distúrbio detectado, verificam-se os seguintes parâmetros: 
1. Em faltas, os valores de $E_{\text {pre }}$ do primeiro PT, de todas as energias $\left(\mathcal{E}_{v_{A}}, \mathcal{E}_{v_{B}}, \mathcal{E}_{v_{C}}, \mathcal{E}_{i_{A}}, \mathcal{E}_{i_{B}}\right.$ e $\left.\mathcal{E}_{i_{C}}\right)$, localizamse na região de regime permanente $\left(E_{1} \leqslant E_{\text {pre }} \leqslant E_{2}\right)$. Estes parâmetros são úteis para distinção entre as faltas e as energizações, visto que para as correntes em uma energização, $E_{p r e}<E_{1}$.

2. Defina $E_{\text {pos }}$ como o valor médio de energia, para cada curva de energia das correntes de fase $\left(\mathcal{E}_{i_{A}}, \mathcal{E}_{i_{B}}\right.$ e $\left.\mathcal{E}_{i_{C}}\right)$, calculado no último ciclo do respectivo sinal. No caso de mais de um distúrbio detectado, $E_{\text {pos }}$ do distúrbio $j$ passa a ser $E_{\text {pre }}$ do primeiro PT do distúrbio $j+1$. Em faltas e em dasligamentos de LTs, $E_{\text {pos }}<E_{1}$ (corrente nula após abertura dos terminais da LT). Os outros distúrbios transitórios normalmente apresentam $E_{1} \leqslant E_{\text {pos }} \leqslant E_{2}$.

3. Defina $\mathcal{N}$ o número de PTs em um distúrbio transitório. Conforme a análise da falta ilustrada na Fig. 2, uma falta é caracterizada por dois ou mais PTs $(\mathcal{N} \geqslant 2)$, relacionados aos instantes inicial e final. Por outro lado, desligamentos de LTs apresentam apenas um PT $(\mathcal{N}=1)$.

Conforme o exposto, em uma falta tem-se $E_{\text {pos }}<E_{1}$. No entanto, devido ao decaimento dc com ruídos nas correntes do secundário do TC em registros oscilográficos reais (seção 2.1.6), $E_{\text {pos }}$ referente a cada corrente atinge esta condição após alguns ciclos pós-falta. Na Fig. 3, não é possível visualizar $E_{\text {pos }}$ referentes às correntes, pois apenas quatro ciclos de energias pós-falta são ilustrados.

\section{MÉTODO PROPOSTO}

Os métodos de detecção e localização dos instantes de falta propostos neste artigo estão inseridos em um método de diagnóstico de distúrbios, descrito a seguir:

- Detecção de faltas: Inicialmente, aplica-se um estágio da TWD aos sinais de tensão e corrente de fase, obtendo-se os coeficientes wavelet da primeira escala. Calculam-se as energias janeladas dos coeficientes wavelet de cada sinal através da equação (3), com $j=1$. Em seguida, normalizam-se as curvas de energias. A detecção dos transitórios é realizada através da análise das curvas de energias dos coeficientes wavelet das tensões e correntes, via um aumento brusco de energia. Por fim, o método verifica se os transitórios detectados são referentes a uma falta ou a um outro distúrbio transitório.

- Localização dos instantes de ocorrência dos transitórios: Esta etapa é baseada na análise dos coeficientes wavelet das tensões e correntes de fase, da primeira escala, em meio ciclo após a detecção de cada PT.

- Classificação de faltas: Além da detecção e identificação das faltas, as energias janeladas dos coeficientes wavelet também são utilizadas na classificação de faltas. Este módulo não é tratado neste artigo.
Um resumo do método de diagnóstico de faltas proposto é ilustrado na Fig. 4.

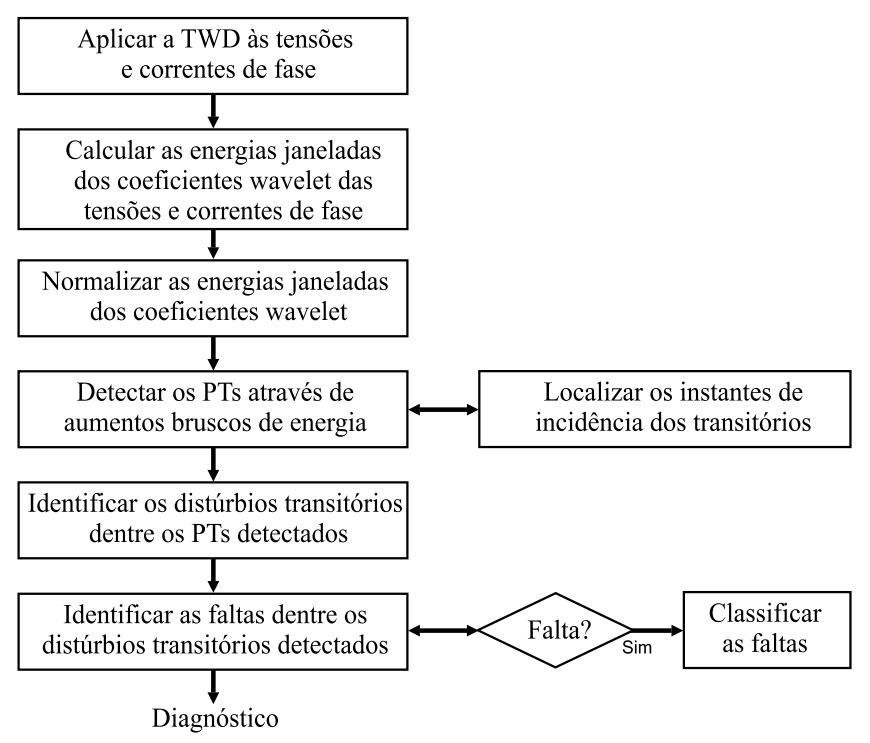

Figura 4: Fluxograma resumido do método proposto.

\section{DETECÇÃO DE FALTAS}

Considere $k$ uma determinada amostra nas curvas de energia $\left(\mathcal{E}_{v_{A}}, \mathcal{E}_{v_{B}}, \mathcal{E}_{v_{C}}, \mathcal{E}_{i_{A}}, \mathcal{E}_{i_{B}}\right.$ e $\left.\mathcal{E}_{i_{C}}\right)$ e $i$ um PT. Apresentam-se a seguir as regras utilizadas na detecção dos PTs:

1. Faça $k=1$ e $i=0$.

2. Se $k \leqslant\left(k_{f}-W\right) / 2$ (análise até o penúltimo ciclo).

(a) A partir de $k$, identifique se existe algum $\Delta E(m) \geqslant 3$, para as energias $\mathcal{E}_{v_{A}}, \mathcal{E}_{v_{B}}, \mathcal{E}_{v_{C}}, \mathcal{E}_{i_{A}}$, $\mathcal{E}_{i_{B}}$ e $\mathcal{E}_{i_{C}}$.

(b) Se nenhum $\Delta E(m) \geqslant 3$ foi encontrado, então termine as análises.

(c) Faça $i=i+1, k_{i E}=2 m$ e calcule $E_{\text {pre }}$ nas energias $\mathcal{E}_{v_{A}}, \mathcal{E}_{v_{B}}, \mathcal{E}_{v_{C}}, \mathcal{E}_{i_{A}}, \mathcal{E}_{i_{B}}$ e $\mathcal{E}_{i_{C}}$.

(d) Utilize as regras de localização dos transitórios, propostas na próxima seção, para obter $k_{i C}{ }^{1}$.

(e) Faça $k=k_{i E} / 2+W / 4$ (adianta $1 / 2$ ciclo) e volte ao passo 2 para detecção de outros PTs.

Após a detecção dos PTs, o método proposto identifica quais PTs pertencem a um determinado distúrbio. Todos os distúrbios a princípio são classificados como distúrbios transitórios. Em seguida, o método identifica quais distúrbios são referentes a uma falta. Apresentam-se a seguir as regras utilizadas na detecção das faltas:

\footnotetext{
${ }^{1} \mathrm{O}$ instante inicial de um PT $i\left(k_{i}\right)$ obtido através de um aumento brusco de energia é $k_{i E}$. No entanto, $k_{i E} \leqslant k_{i C} \leqslant k_{i E}+W / 2$ é a estimação desse instante obtida pelo método de localização dos transitórios.
} 
1. Se mais de um PT foi detectado $(\mathcal{N}>1)$, então a partir do segundo PT, verifique:

(a) Se o $i$-ésimo PT apresenta $E_{p r e}>E_{2}$ em pelo menos uma das curvas de energias $\mathcal{E}_{v_{A}}, \mathcal{E}_{v_{B}}, \mathcal{E}_{v_{C}}$, $\mathcal{E}_{i_{A}}, \mathcal{E}_{i_{B}}$ e $\mathcal{E}_{i_{C}}$. Neste caso, os PTs $i$-1 e $i$ pertencem ao mesmo distúrbio.

(b) Senão, o $i$-ésimo PT é o primeiro PT de um novo distúrbio.

2. Calcule $E_{\text {pos }}$ de cada distúrbio conforme a seção 3.2.

3. Um determinado distúrbio transitório é uma falta se:

(a) No primeiro PT do distúrbio, $E_{\text {pre }}>E_{1}$ em todas as energias $\mathcal{E}_{v_{A}}, \mathcal{E}_{v_{B}}, \mathcal{E}_{v_{C}}, \mathcal{E}_{i_{A}}, \mathcal{E}_{i_{B}}$ e $\mathcal{E}_{i_{C}}$.

(b) $E_{\text {pos }}<E_{1}$ em pelo menos uma das energias $\mathcal{E}_{I A}$, $\mathcal{E}_{I B}$ e $\mathcal{E}_{I C}$.

(c) $\mathcal{N} \geqslant 2$.

\section{LOCALIZAÇÃO DOS INSTANTES DE FALTA}

$\mathrm{O}$ instante inicial do $i$-ésimo PT é definido pela amostra $k_{i}$. O método de detecção de transitórios, no entanto, identifica a localização do $i$-ésimo PT na amostra $k_{i E}$ quando um aumento brusco de energia é detectado. Normalmente, $k_{i E}$ é uma boa estimação de $k_{i}$. Entretanto, tem-se um erro devido as cinco amostras necessárias à detecção de um aumento brusco de energia. Como alternativa, o método de localização identifica nos coeficientes wavelet, a partir de $k_{i E}$, uma estimativa mais precisa da $k_{i}$, denominada de $k_{i C}$.

Análise do estado da arte mostrou que nos métodos baseados na TWD para localização de transitórios, os coeficientes wavelet relacionados aos transitórios possuem amplitude absoluta elevada e próxima ao valor máximo, enquanto que os coeficientes relacionados aos ruídos no regime permanente possuem amplitude baixa, podendo ser facilmente eliminados por meio de uma etapa de filtragem (Santoso et al., 1997; Costa et al., 2006a), que consiste em descartar os coeficientes com amplitude inferior a uma certa percentagem do valor máximo. Após a filtragem, o primeiro e último coeficiente de cada sinal indicam os instantes inicial e final dos distúrbios, respectivamente. No caso das faltas, entretanto, a estimativa desses instantes torna-se mais complexa devido a alguns fatores, como por exemplo, os pré-transitórios e a abertura dos terminais da LT em diferentes instantes.

A complexidade deste problema pode ser melhor ilustrada usando-se o exemplo mostrado na Fig. 5, na qual apresentam-se os coeficientes wavelet das tensões e correntes de fase, da primeira escala, do registro oscilográfico real ilustrado na Fig. 2.

Conforme ilustrado na Fig. 5, os coeficientes wavelet de $v_{A}$ e $v_{B}$ indicam, com boa precisão, o instante inicial da falta $\left(k_{2} / 2\right)$. No entanto, os coeficientes relacionados ao instante final da falta $\left(k_{3} / 2\right)$ possuem baixa amplitude quando comparados aos coeficientes no instante inicial da falta, sendo facilmente eliminados na etapa de filtragem. Por outro lado, os coeficientes wavelet de $v_{C}$ são mais apropriados, neste caso, na estimação do instante de abertura do terminal remoto da LT $\left(k_{4} / 2\right)$, sendo os coeficientes relacionados aos instantes inicial e final da falta, em $k_{2} / 2$ e $k_{3} / 2$, eliminados na filtragem.

Com relação às correntes, os coeficientes wavelet das correntes de falta $\left(i_{A}\right.$ e $\left.i_{B}\right)$ a partir das oscilações pré-falta, em $k_{1} / 2$, até o instante em que o terminal remoto foi aberto, em $k_{4} / 2$, possuem magnitude em valor absoluto da mesma ordem de grandeza dos coeficientes relacionados aos instantes inicial e final da falta, em $k_{2} / 2$ e $k_{3} / 2$, respectivamente. Isto se deve à natureza da falta, que propiciou a incidência de componentes de alta frequência durante todo o distúrbio. Como consequência, a localização dos instantes inicial e final da falta através desses coeficientes é impraticável na primeira escala. No entanto, os coeficientes wavelet da corrente da fase $\mathrm{C}$ sofrem alterações abruptas após o instante inicial da falta devido ao acoplamento eletromagnético entre as fases, podendo ser utilizados com sucesso na estimação do instante inicial da falta, em $k_{2} / 2$. A localização do instante final da falta $\left(k_{3} / 2\right)$ depende do valor do limiar na filtragem.

Para se obter uma estimativa mais precisa dos instantes de incidência dos transitórios, o método utiliza os coeficientes wavelet das tensões e correntes em apenas meio ciclo após cada PT $i$ detectado, ou seja, em meio ciclo após $k_{i E}$.

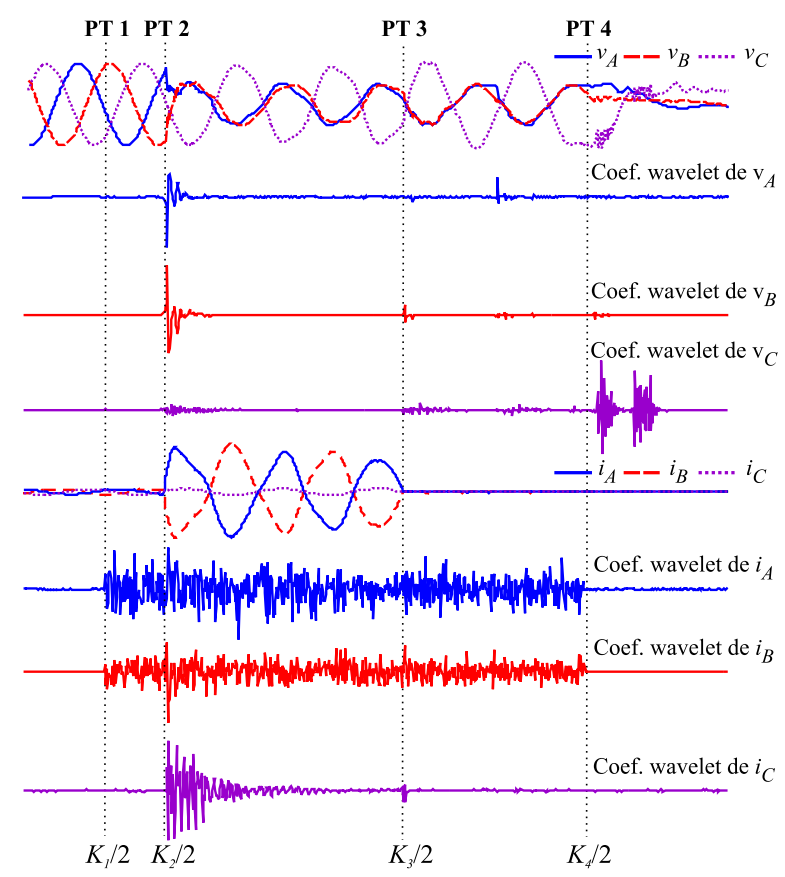

Figura 5: Coeficientes wavelet, da primeira escala, das tensões e correntes de uma falta real do tipo $A B$. 
Apresentam-se na Fig. 6 os coeficientes wavelet normalizados da tensão na fase $\mathrm{B}$, da primeira escala, normalizadas em meio ciclo após os PTs 2, 3 e 4, destacando-se as amostras $k_{i}$ (instante de ocorrência do $i$-ésimo PT) e as respectivas estimativas $k_{i E}$ (amostras obtidas na etapa de detecção). Constatou-se que as amostras relacionadas aos diversos PTs podem ser facilmente localizadas a partir das amostras $k_{i E}$.

\subsection{Filtragem dos Coeficientes Wavelet}

Conforme seção 3, os coeficientes wavelet da primeira escala são apropriados para detecção e localização das componentes de alta frequência oriundas dos transitórios. Entretanto, os sinais oscilográficos reais normalmente apresentam ruídos que podem dificultar a localização correta dos transitórios. A eliminação dos coeficientes referentes aos ruídos pode ser feita através de técnicas de filtragem baseadas no valor absoluto dos coeficientes wavelet (Chen et al., 2003).

As técnicas de filtragem tradicionais consistem em eliminar os coeficientes wavelet cujas amplitudes em valor absoluto, sejam inferiores a um certo limiar $L$. Destaca-se aqui a técnica de filtragem, denominada de hard wavelet thresholding (HWT) (Santoso et al., 1997), na qual o valor do limiar é baseado no máximo valor absoluto dos coeficientes wavelet $(\max (||)$.$) , entre as amostras k_{m}$ a $k_{n}$, como segue:

$$
L\left(k_{m}, k_{n}\right)=U \cdot \max \left(\left|d\left(k_{m}, k_{n}\right)\right|\right),
$$

sendo $d\left(k_{m}, k_{n}\right)$ os coeficientes wavelet da primeira escala, entre as amostras $k_{m}$ a $k_{n} ; 0 \leq U<1$. Por exemplo, para $U=0,15$ os coeficientes cujas amplitudes em valores absolutos sejam inferiores a $15 \%$ da maior amplitude, também em valor absoluto, serão anulados conforme a seguinte equação:

$$
\hat{d}(k)=\left\{\begin{array}{l}
d(k), \text { se }|d(k)| \geqslant L\left(k_{m}, k_{n}\right), \\
0, \text { caso contrário }
\end{array}\right.
$$

sendo $k_{m} \leqslant k \leqslant k_{n}$ e $\hat{d}$ os coeficientes wavelet da primeira escala após o processo de filtragem.

Esse processo é ilustrado na Fig. 7, na qual apresentam-se os coeficientes wavelet normalizados da tensão na fase $\mathrm{B}$, da primeira escala, após uma filtragem com $U=0,4$, em meio ciclo após os PT 2, 3 e $4 . k_{i C_{-} V B}$ é o instante estimado do $i$-ésimo PT através da análise dos coeficientes wavelet da tensão da fase B. $k_{i C_{-} V B} / 2$ passa a ser o primeiro coeficiente wavelet diferente de zero após $k_{i E} / 2$.

\subsection{Sumário da Etapa de Localização dos Transitórios}

$\mathrm{O}$ instante inicial do $i$-ésimo PT $\left(k_{i C}\right)$ é obtido através dos instantes inicial das tensões e correntes de fase $\left(k_{i C_{-} V A}\right.$, $k_{i C_{-} V B}, k_{i C_{-} V C}, k_{i C_{-} I A}, k_{i C_{-} I B}$ e $\left.k_{i C_{-} I C}\right)$ referentes ao respectivo PT. A seguir, apresentam-se as regras utilizadas na localização do $i$-ésimo PT:

1. Aplique a técnica de filtragem HWT $(U=0,4)$ aos coefi- cientes wavelet de $v_{A}$, entre $k_{i E} / 2$ e $k_{i E} / 2+W / 4$.

2. Identifique o índice do primeiro coeficiente wavelet diferente de zero $\left(k_{i C_{-} V A}\right)$.

3. Repita os passos 1 e 2 para as tensões $v_{B}$ e $v_{C}$ e correntes $i_{A}, i_{B}$ e $i_{C}$, obtendo os instantes iniciais $k_{i C_{-} V B}$, $k_{i C_{-} V C}, k_{i C_{-} I A}, k_{i C_{-} I B}$ e $k_{i C_{-} I C}$, respectivamente.

4. O instante inicial do $i$-ésimo PT $\left(k_{i C}\right)$ passa a ser o valor mais frequente entre $k_{i C_{-} V A}, k_{i C_{-} V B}, k_{i C_{-} V C}, k_{i C_{-} I A}$, $k_{i C_{-} I B}$ e $k_{i C_{-} I C}$. Caso eles sejam todos diferentes, então $k_{i C}$ passa a ser o maior valor entre eles.

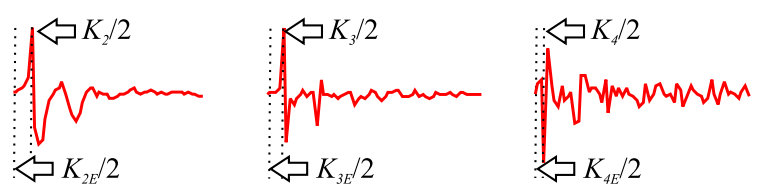

Figura 6: Coeficientes wavelet normalizados em meio ciclo, da primeira escala, de $v_{B}$ após os PTs 2, 3 e 4.

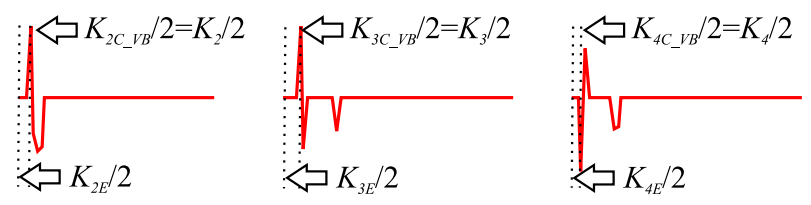

Figura 7: Coeficientes wavelet normalizados e filtrados em meio ciclo, da primeira escala, de $v_{B}$ após os PTs 2, 3 e 4.

\section{AVALIAÇÃO DO MÉTODO PROPOSTO}

\subsection{Registros Oscilográficos Simulados}

O ângulo de incidência, resistência e distância da ocorrência das faltas são parâmetros que influenciam a natureza dos transitórios em um curto-circuito. Para se avaliar a influência desses parâmetros, simulações digitais foram realizadas em um programa do tipo EMTP, o Alternative Transient Program (ATP) (Leuven EMTP Center, 1987). Utilizou-se o modelo de um sistema elétrico de $230 \mathrm{kV}$ (Fig. 8), proposto por IEEE Power System Relaying Committee (2004) para o teste de algoritmos de proteção.

O modelo do sistema elétrico é composto por: duas fontes ideais $\mathrm{S} 1$ e $\mathrm{S} 3$; uma máquina síncrona $\mathrm{S} 2$ conectada na barra 4 por meio de um transformador $Y-\triangle$, com Y aterrado; uma LT entre as barras 2 e 4 (LT4); uma LT de circuito duplo entre as barras 1 e 2 (LT1 e LT2), a partir da qual emana uma LT até a barra 3 (LT3). Os transformadores para instrumento também fazem parte do modelo (IEEE Power System Relaying Committee, 2004). O modelo original foi adaptado para comportar um RDP em cada terminal das LTs, com taxa de amostragem de $15360 \mathrm{~Hz}$.

As faltas foram simuladas na seção L2T1, da LT2 (Fig. 8), com $r=50 \Omega$, obtendo-se um registro da ocorrência no RDP1 
(no terminal próximo ao distúrbio) e no RDP2 (no terminal mais distante do distúrbio). Todos os tipos de falta: AT, BT, $\mathrm{CT}, \mathrm{AB}, \mathrm{BC}, \mathrm{AC}, \mathrm{ABT}, \mathrm{BCT}, \mathrm{ACT}$ e ABC foram simulados, com ângulo de incidência $\theta_{o}=\left\{0^{\circ}, 10^{\circ}, 20^{\circ}, \ldots, 180^{\circ}\right\}$. Desta forma, além da distância de falta e do ângulo de incidência, o tipo de falta também foi avaliado nas simulações.

De um total de 380 registros simulados com faltas, o PT relacionado à incidência da falta em 8 registros oscilográficos não foram detectados, enquanto que o PT relacionado ao instante final da falta foi detectado em todos os casos. Portanto, o método de detecção de distúrbios transitórios apresentou uma taxa de acerto de $100 \%$, sendo detectado falta em $97,89 \%$ dos registros oscilográficos.

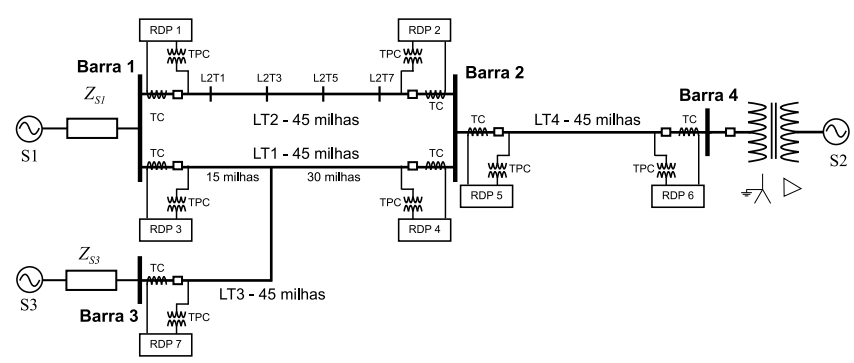

Figura 8: Modelo de um sistema elétrico de $230 \mathrm{kV}$ proposto pelo IEEE, utilizado para a simulação das faltas.

Com relação à localização dos transitórios, o erro correspondente à estimação do $i$-ésimo PT, em ms, é calculado por:

$$
e=\left|k_{i}-k_{i C}\right| / f_{s}
$$

sendo $k_{i}$ o instante de ocorrência dos transitórios; $k_{i C}$ o instante obtido pelo método; $f_{s}$ a frequência de amostragem.

Tomando-se como referência 1 ciclo $(16,66 \mathrm{~ms})$, o método de localização dos transitórios apresentou resultados satisfatórios, visto que foram obtidos erros médios de 0,07 e 0,48 ms na estimação dos instantes inicial e final das faltas, respectivamente.

\subsection{Registros Oscilográficos Reais}

Para validar o desempenho do método proposto, utilizaramse 1692 registros oscilográficos reais, obtidos em diversas partes do sistema de potência da Chesf, no período de 2003 a 2008, contemplando os níveis de tensão de 138, 230 e 500 $\mathrm{kV}$, com frequências de amostragem de 1200 e 15360 Hz (Tabela 1). Diversos tipos de distúrbios foram avaliados: faltas, faltas evolutivas, afundamentos de tensão, múltiplos distúrbios, etc.

Conforme a Tabela 1, o método de detecção apresentou resultados satisfatórios na análise dos 1696 registros, visto que apenas 6 distúrbios transitórios não foram detectados.
Tabela 1: Desempenho do método de detecção de faltas diante dos registros oscilográficos reais.

\begin{tabular}{|c|c|c|c|}
\hline Registro oscilográfi co & Quantidade & Diagnóstico obtido & Quantidade \\
\hline Sem distúrbios & 905 & Sem distúrbios & 905 \\
\hline Falta & 59 & $\begin{array}{c}\text { Falta } \\
\text { Dist. Transitório }\end{array}$ & $\begin{array}{c}58 \\
1\end{array}$ \\
\hline Falta Evolutiva & 6 & Falta & 6 \\
\hline Falta + Religamento & 5 & $\begin{array}{c}\text { Falta }+ \\
\text { Dist. Transitório }\end{array}$ & 5 \\
\hline Afund. de tensão & 219 & Dist. Transitório & 219 \\
\hline $\begin{array}{l}\text { Afund. de tensão + } \\
\text { Variação de carga }\end{array}$ & 22 & $\begin{array}{l}\text { Dist. Transitório + } \\
\text { Dist. Transitório }\end{array}$ & 22 \\
\hline $\begin{array}{l}\text { Afund. de tensão + } \\
\text { Afund. de tensão }\end{array}$ & 20 & $\begin{array}{l}\text { Dist. Transitório + } \\
\text { Dist. Transitório }\end{array}$ & 20 \\
\hline Trans. de chaveamento & 175 & Dist. Transitório & 175 \\
\hline Energização de LT & 118 & $\begin{array}{l}\text { Dist. Transitório } \\
\text { Sem distúrbios }\end{array}$ & $\begin{array}{c}116 \\
2\end{array}$ \\
\hline $\begin{array}{c}\text { Dupla Energização } \\
\text { de LT }\end{array}$ & 1 & $\begin{array}{l}\text { Dist. Transitório + } \\
\text { Dist. Transitório }\end{array}$ & 1 \\
\hline Desligamento de LT & 97 & $\begin{array}{l}\text { Dist. Transitório } \\
\text { Sem distúrbios }\end{array}$ & $\begin{array}{c}93 \\
4\end{array}$ \\
\hline Energização de BC & 35 & Dist. Transitório & 35 \\
\hline Desligamento de BC & 34 & Dist. Transitório & 34 \\
\hline
\end{tabular}

De um total de 70 registros oscilográficos reais com falta avaliados, independente do nível de tensão e da taxa de amostragem, apenas uma falta não foi detectada. Análise manual do registro constatou que a corrente de falta aumentou progressivamente, com transitórios bastante amortecidos. O tempo total da falta foi de aproximadamente 14 ciclos. Apenas o PT relacionado ao instante final da falta foi detectado, sendo o evento classificado como distúrbio transitório, ao invés de falta.

As faltas evolutivas foram corretamente detectadas, com os instantes em que a falta mudou de tipo devidamente identificados. Todos os distúrbios em registros oscilográficos com múltiplos distúrbios foram devidamente detectados.

O método de localização dos transitórios apresentou um erro médio de aproximadamente 0,32 e 1,44 ms na obtenção dos instantes inicial e final das faltas, respectivamente. Na Tabela 2 apresentam-se os resultados obtidos na localização dos transitórios de falta, tomando-se como base o valor de $1 \mathrm{~ms}$ (aproximadamente $1 / 16$ ciclo para $f=60 \mathrm{~Hz}$ ).

Tabela 2: Resultados da localização dos instantes de falta.

\begin{tabular}{ccccc}
\hline \multicolumn{2}{c}{ Instante inicial da falta } & & \multicolumn{2}{c}{ Instante fi nal da falta } \\
\hline Regist. avaliados (\%) & erro & & Regist. avaliados (\%) & erro \\
\hline \hline 94,65 & $\leqslant 1 \mathrm{~ms}$ & 73,21 & $\leqslant 1 \mathrm{~ms}$ \\
5,35 (três registros) & $>1 \mathrm{~ms}$ & 26,79 & $>1 \mathrm{~ms}$ \\
\hline
\end{tabular}


A principal dificuldade na identificação correta do instante inicial das faltas reais avaliadas foi a presença dos prétransitórios nas correntes de falta (Fig. 2). De um total de 70 registros oscilográficos com falta avaliados, 33 apresentaram pré-transitórios. Entretanto, em apenas três registros foram obtidos erro acima de $1 \mathrm{~ms}$.

Com relação ao instante final da falta, o erro médio acima de $1 \mathrm{~ms}$ encontrado está relacionado à dificuldade da localização correta do instante da abertura dos pólos do disjuntor.

\section{CONCLUSÃO}

Um método baseado na transformada wavelet para detecção de faltas e distúrbios transitórios foi apresentado. O desempenho do método foi avaliado utilizando-se registros oscilográficos simulados e reais obtidos de várias linhas de transmissão do sistema Chesf.

A principal contribuição do método é a sua generalização atribuída ao esquema de normalização adotado, visto que as análises dos registros oscilográficos independem dos diversos níveis de tensão, da topologia do sistema e das taxas de amostragem dos registradores digitais de perturbações da Chesf. O método apresentou bons resultados ao ser avaliado com registros reais com falta, afundamento de tensão, transitórios de chaveamento, energização e desligamento de linhas de transmissão e de banco de capacitores. Registros com faltas evolutivas e múltiplos distúrbios também foram avaliados pelo método proposto e bons resultados também foram obtidos.

A etapa de detecção dos distúrbios transitórios apresentou resultados satisfatórios. Dos 1696 registros reais avaliados, o método não detectou distúrbios transitórios em apenas 6 registros. A etapa de localização dos transitórios também foi bem sucedida, apresentando um erro médio de aproximadamente 0,3 e 1,4 ms na localização dos instantes inicial e final das faltas, respectivamente.

Ao final, constatou-se que a energia janelada dos coeficientes wavelet constitui-se em uma ferramenta poderosa na detecção, identificação e localização no tempo dos distúrbios transitórios.

\section{AGRADECIMENTOS}

Os autores agradecem ao Conselho Nacional de Desenvolvimento Científico e Tecnológico (CNPq), Fundação de Apoio à Pesquisa do Estado da Paraíba (FAPESQ) e a Companhia Hidro Elétrica do São Francisco (Chesf) pelos suportes financeiro e técnico.

\section{REFERÊNCIAS}

Anderson, P. M. (1999). Power System Protection, IEEE Press Series on Power Engineering, Piscataway, NJ USA.

ANEEL (2007). Resolução Normativa No. 270.

Bollen, M. H. J. (2000). Understanding Power Quality Problems, IEEE, New York, USA.

Bollen, M. H. J. and Gu, I. Y.-H. (2006). Signal Processing of Power Quality Disturbances, IEEE, New York, USA.

Bradley, A. D., Gray, C. B. and O'Kelly, D. (1978). Transient compensation of current transformers, IEEE Transaction on Power Apparatus and Systems PAS97(4): 1264-1271.

Chen, W., Malik, O. P., Yin, X., Chen, D. and Zhang, Z. (2003). Study of wavelet-based ultra high speed directional transmission line protection, IEEE Transactions on Power Delevery 18(4): 1134-1139.

Costa, F. B., Silva, K. M., Dantas, K. M. C., Souza, B. A. and Brito, N. S. D. (2006a). A wavelet-based method for fault clearing time delimitation, Congresso Brasileiro de Automática pp. Salvador-BA, Brasil.

Costa, F. B., Silva, K. M., Dantas, K. M. C., Souza, B. A. and Brito, N. S. D. (2006c). A method for fault classification in transmission lines based on ann and wavelet coefficients energy, VII World Congress on Computational Inteligency pp. Vancouver, Canada.

Costa, F. B., Silva, K. M., Dantas, K. M. C., Souza, B. A. and Brito, N. S. D. (2007). A wavelet-based algorithm for disturbances detection using oscillographic data, International Conference on Power Systems Transients pp. Lyon, France.

Costa, F. B., Souza, B. A. and Brito, N. S. D. (2008). A wavelet-based algorithm to analyze oscillographic data with single and multiple disturbances, IEEE PES General Meeting pp. Pittsburgh, USA.

Costa, F. B., Souza, B. A., Brito, N. S. D., Silva, K. M. and Dantas, K. M. C. (2006b). A wavelet-based algorithm for fault classification using oscillographic data, International Conference on Harmonics and Quality of Power pp. Cascais, Portugal.

Daubechies, I. (1992). Ten Lectures on Wavelets, CBMSNSF Regional Conference Series, SIAM, Philadelphia, USA. 
Girsis, A. A. and Brown, R. G. (1983). Modelling of faultinduced noise signals for computer relaying applications, IEEE Transactions on Power Apparatus and Systems PAS-102(9): 2834-2841.

Hong, Y. Y. and Chang-Chian, P. C. (2008). Detection and correction of distorted current transformer current using wavelet transform and artificial intelligence, IET Gener. Transm. Distrib. 2(4): 566-575.

IEEE Power Engineering Society (2003). IEEE Guide for Automatic Reclosing of Line Circuit Breakers for AC Distribution and Transmission Lines - IEEE Std C37.104-2002, New York, USA.

IEEE Power System Relaying Committee (2004). EMTP Reference Models for Transmission Line Relay Testing, <http://www.pes-psrc.org/Reports-/d6report.zip>.

Kang, Y. C., Lim, U. J., Kang, S. H. and Crossley, P. A. (2004). Compensation of the distortion in the secondary current caused by saturation and remanence in a ct, IEEE Transactions on Power Delivery 19(4): 16421649 .

Leuven EMTP Center (1987). ATP - Alternative Transient Program - Rule Book, Herverlee, Belgium.

Li, F., Li, Y. and Aggarwal, R. K. (2002). Combined wavelet transform and regression technique for secondary current compensation of current transformers, IEE Proc.Gener. Transm. Distrib. 149(4): 497-503.

Mallat, S. G. (1989). A theory for multiresolution signal decomposition: The wavelet representation, IEEE Transaction on Pattern Analysis and Machine Intelligence 11(7).

Oleskovicz, M., Coury, D. V., Carneiro, A. A. F. M., Arruda, E. F., Filho, O. D. and Souza, S. A. (2006). Estudo comparativo de ferramentas modernas de anÁlise aplicadas À qualidade da energia elÉtrica, Controle e Automação 17(3): 331-341.

Oppenheim, A. V. and Schafer, R. W. (1989). Discrete-time signal processing, pp. New Jersey, USA.

Robertson, D. C., Camps, O. I. and Gish, J. S. M. W. B. (1996). Wavelets and electromagnetic power system transients, IEEE Transaction on Power Delivery 11(2): 1050-1058.

Santoso, S., Powers, E. J. and Grady, W. M. (1997). Power quality disturbance data compression using wavelet transform methods, IEEE Transactions on Power Delivery 12(3): 1250-1256.
Santoso, S., Powers, E. J., Grady, W. M. and Hofmann, P. (1996). Power quality assessment via wavelet transform analysis, IEEE Transactions on Power Delivery 11(2): 924-930.

Silva, K. M., Souza, B. A., Brito, N. S. D., Dantas, K. M. C., Costa, F. B. and Silva, S. S. B. (2007). Detecção e classificação de faltas a partir da análise de registros oscilográficos via redes neurais artificiais e transformada wavelet, Controle e Automação 18(2): 163-172.

Silveira, P. M., Seara, R. and Zurn, H. H. (2002). Seleção de fases em relás numéricos de linhas de transmissão baseada em análise multi-resolução via trasnformada wavelet, Controle e Automação 13(3): 327-336.

Summary Update of Practices on Breaker Failure Protection (1982). IEEE Transactions on Power Apparatus and Systems PAS-101(3): 555-563.

Swift, G. W. (1979). The spectra of fault-induced transients, IEEE Transactions on Power Apparatus and Systems PAS-98(3): 940-947.

Zaneta Jr., L. C. (2003). Trasitórios Eletromagnéticos em Sistemas de Potência, Editora da Universidade de São Paulo, São Paulo, Brasil. 